\title{
Pregnancy and the immune system: between tolerance and rejection
}

\author{
Olivier Thellin, Ernst Heinen* \\ Institute of Human Histology, University of Liège, 20 Rue de Pitteurs, B-4020 Liège, Belgium
}

\begin{abstract}
Interactions between the conceptus and the mother are bi-directional: the feto-placental tissues need nutrition and a suitable environment in homeostatic condition whereas the mother influenced by the placental factors adapts her metabolism and immune system. Many different mechanisms acting locally or at distance ensure tolerance of the semiallogeneic graft by the maternal natural and adaptive immune defences. In front of this tolerance, mechanisms exist ensuring rejection of the conceptus by the mother (spontaneous abortion) through rupture of one or more tolerance mechanisms, notably in stress situations endangering the mother. Thus outcome of a pregnancy is dependent on efficiently working tolerance mechanisms, and rupture of such mechanisms can lead to rejection. The balance of influence leading either to tolerance or rejection is under control of internal (maternal and fetal) and external (environmental) factors. Rejection, if triggered, mainly occurs through immune-induced inflammation, tissue degradation and coagulation.
\end{abstract}

(C) 2002 Elsevier Science Ireland Ltd. All rights reserved.

Keywords: Pregnancy; Immune system; Tolerance

\section{Between tolerance and rejection}

An embryo is a mating product of histoincompatible individuals in an outbred population. It can be compared with a semi-allogeneic graft which has to be tolerated over all the gestational period (for review see Thellin et al., 2000).

* Corresponding author. Tel.: +32-4-366-5170; fax: +32-4366-5173

E-mail address: eheinen@ulg.ac.be (E. Heinen).
Intimate contacts between the foetus and the maternal tissues ensure nutrition of the fetus and adaptation of the mother to this graft. The placenta, composed of fetal and maternal structures, is both a barrier and a zone for intense exchanges. On one hand, due to the paternal antigens, which the fetus is susceptible to express, the maternal immune system risks at each time to induce rejection. The maternal immune system is aware of the presence of the fetus and the placenta and can be induced to reject this graft (Erlebacher, 2001). Thus tolerance mechanisms have evolved during evolution to insure the maintenance of the 
feto-placental graft. On the other hand, evolution has retained means to reject the fetus in case of stress endangering the mother, for example, during a severe infectious condition.

In consequence, the feto-placental graft is tolerated or rejected by the maternal immune system. Besides any fetal inadequacy, the decision is notably dependent on environmental parameters, namely on the presence of toxic substances, infectious agents or physical or psychological aggressions.

\section{Means of tolerance}

A lot of hypotheses to explain fetal tolerance have been developed and investigated for 50 years. The mechanisms of fetal tolerance are plural and working at different periods of time in embryonic and fetal development. These tolerance mechanisms act at distance on the maternal immune system or locally at the placental level.

Systemic effects are due to immuno-active hormones, but also fetal antigens and cells circulating in the maternal blood vessels. Indeed, progesterone, an hormone synthesized in large quantities by the placenta, is able to lower immune responses (Siiteri and Stites, 1982). Other hormones, such as the placental growth hormone which replaces progressively the pituitary growth hormone during pregnancy (Hennen et al., 1985), might also help to modulate the immune system (Thellin et al., 2000). Anergy, also called peripheral tolerance, might be induced by fetal antigens or whole cells passing into the maternal blood circulation and the peripheral lymph organs. Indeed, in absence of inflammatory signals such antigens can act as tolerogens.

Locally at the placental level, many different mechanisms are involved, working alone or in interaction with each other. Major histocompatibility complex (MHC) class I expression is reduced on the syncytiotrophoblast. Its specific expression of human leukocyte antigen-G (HLAG) may block the cytolytic activity of natural killer (NK) cells (Moreau et al., 1998), (Fig. 1). In addition to the low MHC expression, antigens could be masked by camouflage systems (Thellin

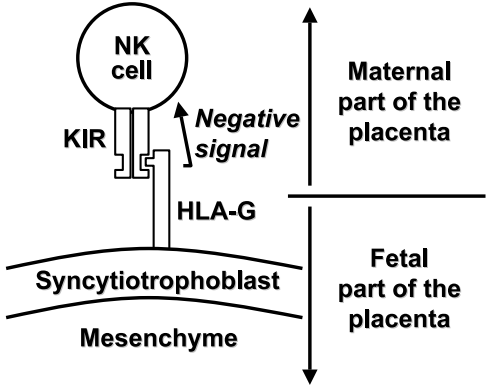

Fig. 1. HLA-G. HLA-G may inhibit cytolytic activity of NK cells by binding their KIR receptors (modified from Thellin et al., 2000)
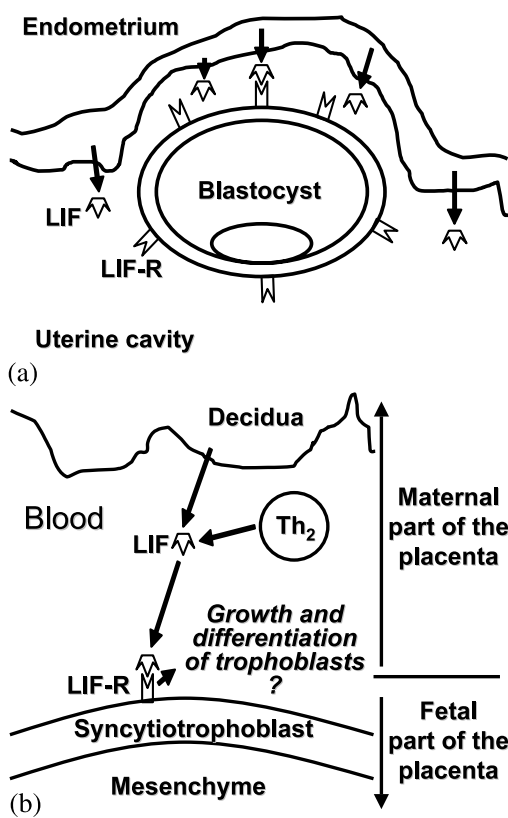

Fig. 2. LIF. (a) LIF (by endometrium) and its receptor (on blastocyst) are essential for implantation. (b) After implantation, LIF could influence the growth and differentiation of trophoblasts (modified from Thellin et al. 2000).

et al., 2000). Leukemia inhibitory factor (LIF) and its receptor are essential for implantation and are thought helping trophoblastic growth or differentiation (Fig. 2a, b), (Senturk and Arici, 1998). Complement inhibitory mechanisms are ensured by blocking molecules, such as MCP (membrane complement protein), DAF (decay accelerating factor (Fig. 3), (Johnson et al., 1990; Holmes et al., 1990) or other factors (i.e. Crry in rodents: Xu 


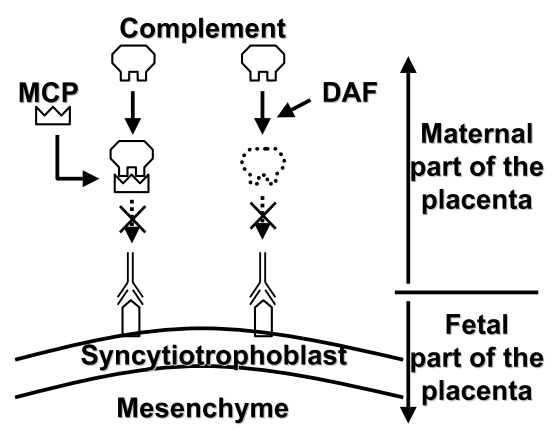

Fig. 3. Complement. Inhibition of complement (by destruction or binding) would reduce its availability to harm fetal tissues (modified from Thellin et al., 2000).

et al., 2000). Indoleamine 2, 3 dioxygenase (IDO) catabolizes tryptophan which is necessary for lymphoid cell activation. It is produced by the syncytiotrophoblast and reduces close-range immune cell reactions (Fig. 4), (Munn et al., 1998). The CD95/CD95-ligand system in involved in cellular turnover, tumor cell elimination, antiviral responses or protection of tissues against activated lymphocytes. It might be active in controlling trophoblastic growth and elimination of cells endangering trophoblastic cells (Fig. 5), Coumans et al., 1999; Runic et al., 1998). This without endangering the trophoblasts themselves. Indeed, CD95 present on trophoblasts does not seem to transduce apoptotic signals in these cells (Payne et al., 1999). Annexin II is secreted by the placenta

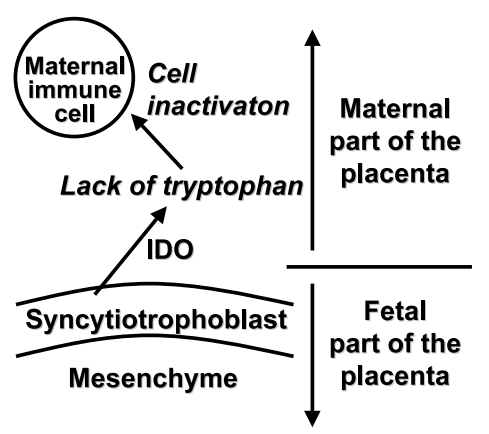

Fig. 4. IDO. IDO is produced by the trophoblast and catabolizes tryptophane at short range and may so inhibit close maternal immune cells (modified from Thellin et al., 2000).

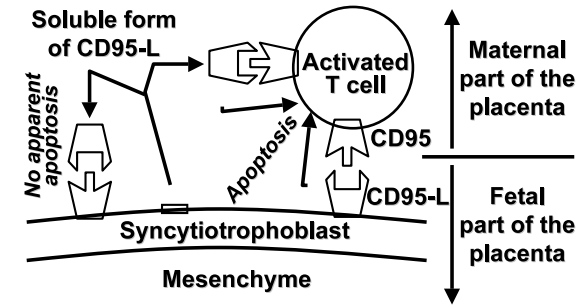

Fig. 5. CD95/CD95-L. Syncytiotrophoblastic CD95-L (membrane or soluble form) can induce apoptosis in maternal immune cells, but not syncytiotrophoblastic cells, expressing CD95 (modified from Thellin et al., 2000).

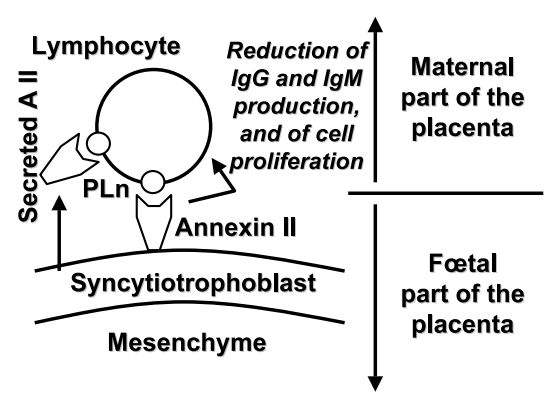

Fig. 6. Annexin II. The Annexin II secreted by the placenta could inhibit lymphoproliferation and secretion of maternal immunoglobulins (modified from Thellin et al., 2000).

and might inhibit lymphoproliferation and secretion of maternal immunoglobulins (Fig. 6), (Aarli and Matre, 1998).

$\mathrm{T}_{\mathrm{h} 1} / \mathrm{T}_{\mathrm{h} 2}$ balance is in favor of $\mathrm{T}_{\mathrm{h} 2}$ cells which produce IL4, IL5, IL6, IL9, IL10, TGF $\beta$ and reduce the production of pro-inflammatory molecules, like IL2 and IFN $\gamma$, which can endanger fetus survival (Raghupathy, 1997; Clark et al., 1999). Recent data presented below suggest that this balance could be a critical and a complex actor in the conceptus tolerance.

The immune cell population at the placental level has also shown some peculiarities. Indeed, the products and surface markers of these cells may differ from generally encountered immune cells of the same types. For example, decidual macrophages are alternatively activated macrophages exerting anti-inflammatory actions. These cells produce little reactive oxygen derivates, but IL10 and IL1-R antagonist (Goerdt and Orfanos, 1999). 
Placental $\mathrm{V} \gamma \delta \mathrm{T}$ cells are activated to produce the immunosuppressive IL10 and TGF $\beta 2$; in humans NK $-\gamma \delta \mathrm{T}$ cells appear to be the main source of TGF $\beta 2$ (Clark et al., 2001b). IL10 and TGF $\beta$ are inhibitors of the Th1 response. The diversity of $\gamma \delta \mathrm{T}$ cells in maternal tissue during pregnancy contrasts with a limited TCR subtype diversity in the non-pregnant state (Bonney et al., 2000; Clark et al., 1999). Many other T, NK and NKT cells are present in the deciduas; the identification of their roles requires further research. These cells also play a part in other fields than tolerance, for example, uterine NK producing interferon gamma contribute to implantation (Ashkar and Croy, 1999). Production of OX-2, a glycoprotein which pushes $\gamma \delta \mathrm{Th}_{1} / \gamma \delta \mathrm{Th}_{2}$ balance toward $\gamma \delta \mathrm{Th}_{2}$, also inhibits macrophages and decreases fgl2, a prothrombinase able to trigger abortion by placental thrombosis and activation of the complement (Fig. 7), (Clark et al., 2001a).

\section{Means for fetal rejection}

By definition, rejection happens when critical tolerance mechanisms are absent or severed.

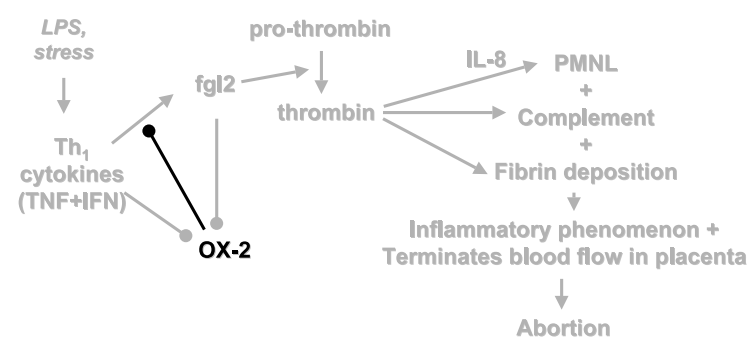

Fig. 7. OX-2 and fgl2. In dark: during normal pregnancy, trophoblastic OX-2 inhibits fgl 2 expression. In grey: when Th1 cytokine expression is activated (by stress or LPS notably), fgl2 expression is increased in the placenta. Fgl2 inhibit OX-2 expression and convert pro-thrombin into thrombin. On one side, thrombin then activates IL-8 synthesis in endothelial cells. IL-8 attracts polymorphonuclear leucocytes (PMNL) leading also to an activation of the complement. On the other side, thrombin triggers clotting via fibrin deposition, terminating the blood flow in placenta. If severe, these events can trigger abortion.
Genotypic or phenotypic alterations can make one or both of the parents unable to conceive, temporarily or definitely. Depending of the alteration, the problem can occur at different steps of the pregnancy, from the fecundation to postimplantation development. The post-implantation rejection is the one most potent to be detected.

While the event initiating rejection may widely vary, most of them could act partly through similar ways. A most important inducible way of rejection involves a $T_{h 1} / T_{h 2}$ imbalance toward $T_{h 1}$, increasing the production of $\mathrm{T}_{\mathrm{h} 1}$ cytokines, notably the pro-inflammatory IFN $\gamma, \mathrm{TNF} \alpha$ and IL2 (Hill et al., 1995). $\mathrm{T}_{\mathrm{h} 1}$ cytokines can activate inflammatory macrophages and NK cells into lectin-activated killers (LAK), which then exert cytolytic capacities. $\mathrm{T}_{\mathrm{h} 1}$ cytokines can also act directly on trophoblastic cells, reducing OX-2 production and increasing their production of fgl 2 (pro-thrombinase). Fgl 2 can then induce clotting, which blocks the circulation of maternal blood in the placenta (thrombosis), thus the vital exchanges between the maternal and fetal bloods. This leads to the death of fetal tissues. Thrombintriggered IL8-production in endothelial cells may attract and activate (Fig. 7) neutrophils, which attack feto-placental structures. $\mathrm{T}_{\mathrm{h} 1}$ cytokines also stimulate the production of immunoglobulins able to activate the complement cascade. Failure to reduce complement capacity contributes then to the abortion process.

This $T_{h 1}$ imbalance can be triggered mainly by infection and stress. Infections leading to a $T_{h 1}$ shift alter syncytiotrophoblastic secretory activities thus reduce the protective tolerance mechanisms. TNF $\alpha$ and IFN $\gamma$, able to act directly on syncytiotrophoblasts, appear to be the effectors inducing spontaneous abortions in stress situations leading to coagulation, arrest of the local maternal blood flow, and aggression of the feto-placental structures (Clark and Croitoru, 2001).

Other factors are also potent to trigger rejection, via $T_{h 1}$ imbalance or not. Hormones or inhibitory molecules can counteract the placental hormones which sustain $\mathrm{T}_{\mathrm{h} 2}$-type immune responses. For example, release of substance $\mathrm{P}$ under stress (and probably other circumstances) from neurons in the uterus induces IL1, TNF $\alpha$ and IFN $\gamma$ production 
(Clark et al., 1999). Toxic substances altering metabolism or vascularization disturb placental development and expressions, thus could decrease or block mechanisms of tolerance, therefore, improve chances of rejection. In consequence, toxic factors risk to alter not only fetal cells, but also maternal functions, namely the tolerance mechanisms.

General modulators of the immune response, for example activators of lymphocytes, macrophages, NK cells, neutrophils, of complement cascade or MHC class I or class II expression may initiate rejection mechanisms. Indeed, the lipopolysaccharides (LPS) released from bacterial membranes during infections are potent activators of immune cells (Arck et al., 1997; Kohmura et al., 2000). Peri-implantation losses in mice, analogous to occult losses in humans, are dependent upon cells producing perforin and complement cascade initiation but not on $\mathrm{TNF} \alpha$ or pro-coagulant activation as observed during abortion of vascularized placenta, analogous to clinically evident human spontaneous miscarriage (Clark et al., 2001b). Thus rejection inducers and effectors differ as related to the evolutionary stage of the conceptus.

\section{Conclusions}

The feto-placental entity has been compared with a graft, a tumor or a parasite in conceptual models (for review see Clark et al., 1999). However, even though mechanisms allowing tolerance can also play roles in graft tolerance, non-rejection of tumors or persistence of parasites, the fetoplacental entity creates its own hormonal ambience profoundly influencing its own or the maternal syntheses. At difference with grafts, tumors or parasites, the feto-placental graft can be discarded if factors endangering the mother dominate, notably through $\mathrm{T}_{\mathrm{h} 1}$ response of the immune system. This finely tuned situation, which on one hand favors conceptus tolerance, but on the other hand allows its rejection, is influenced by internal and external factors. Lots of progress have been accomplished recently in the comprehension of the mechanisms of tolerance or rejection. Current research on the subject will help identify more precisely the factors putting a pregnancy at risk and lead to develop means to circumvent these factors as much as possible.

\section{References}

Aarli, A., Matre, R., 1998. Suppression of immunoglobulin secretion by soluble annexin II. Scand. J. Immunol. 48, $522-526$.

Arck, P., Quarrington, C., Mak, T., Clark, D.A., 1997. TNF- $\alpha$ P55 receptor knockout mice demonstrate two distinct types of endotoxin-induced rejection of the embryo qua parasite. Am. J. Reprod. Immunol. 37, 347.

Ashkar, A.A., Croy, B.A., 1999. Interferon gamma contributes to the normalcy of murine pregnancy. Biol. Reprod. 61, $493-502$.

Bonney, E.A., Pudney, J., Anderson, D.J., Hill, J.A., 2000. Gamma-delta cells in midgestation human pacental villi. Gynecol. Obstet. Invest. 50, 153-157.

Clark, D.A., Arck, P.C., Chaouat, G, 1999. Why did your mother reject you? Immunogenetic determinants of the response to environmental selective pressure expressed at the uterine level. Am. J. Reprod. Immunol. 41, 5-22.

Clark, D.A., Croitoru, K., 2001. TH1/TH2,3 imbalance due to cytokine-producing NK, gammadelta $\mathrm{T}$ and NK-gammadelta $\mathrm{T}$ cells in murine pregnancy decidua in success or failure of pregnancy. Am. J. Reprod. Immunol. 45, 257265.

Clark, D.A., Ding, J.W., Yu, G., Levy, G.A., Gorczynski, R.M., 2001a. Fgl2 prothrombinase expression in mouse trophoblast and deciduas triggers abortion but may be countered by OX-2. Mol. Hum. Reprod. 7, 185-194.

Clark, D.A., Yu, G., Levy, G.A., Gorczynski, R.M., 2001 b. Procoagulants in fetus rejection: the role of the OX-2 (CD200) tolerance signal. Semin. Immunol. 13, 255-263.

Coumans, B., Thellin, O., Zorzi, W., Melot, F., Bougoussa, M., Melen, L., Zorzi, D., Hennen, G., Igout, A., Heinen, E., 1999. Lymphoid cell apoptosis induced by trophoblastic cells: a model of active foeto-placental tolerance. J. Immunol. Methods 224, 185-196.

Erlebacher, A., 2001. Why isn't the fetus rejected? Curr. Opin. Immunol. 13, 590-593.

Goerdt, S., Orfanos, C.E., 1999. Other functions, other genes: alternative activation of antigen-presenting cells. Immunity 10, 137-142.

Hennen, G., Frankenne, F., Closset, J., Gomez, F., Pirens, G., el Khayat, N., 1985. A human placental GH: increasing levels during second half of pregnancy with pituitary GH suppression as revealed by monoclonal antibody radioimmunoassays. Int. J. Fertil. 30, 27-33.

Hill, J.A., Polgar, K., Anderson, D.J., 1995. T-helper 1-type immunity to trophoblast in women with recurrent spontaneous abortion. J. Am. Med. Assoc. 273, 1933-1936. 
Holmes, C.H., Simpson, K.L., Wainwright, S.D., Tate, C.G., Houlihan, J.M., et al., 1990. Preferential expression of the complement regulatory protein decay accelerating factor at fetomaternal interface during human pregnancy. J. Immunol. 144, 3099-3105.

Johnson, P.M., Risk, J.M., Mwenda, J.M., 1990. Human trophoblast expression of retroviral like activity and CD46 (membrane cofactor protein, Hu Ly-m5 and H316 TLX antigen). In: Metler, L., Billington, W.D. (Eds.), Reproductive Immunology. Elsevier, Amsterdam, pp. 125-131.

Kohmura, Y., Kirikae, T., Kirikae, F., Nakano, M., Sato, I., 2000. Lipopolysaccharide (LPS)-induced uterine fetal death (IUFD) in mice is principally due to maternal cause but not fetal sensivity to LPS. Microbiol. Immunol. 44, 897-904.

Moreau, P., Paul, P., Rouas-Freiss, N., Kirszenbaum, M., Dausset, J., Carosella, E.D., 1998. Molecular and immunologic aspects of the nonclassical HLA class I antigen HLAG: evidence for an important role in the maternal tolerance of the fetal allograft. Am. J. Reprod. Immunol. 40, 136144.

Munn, D.H., Zhou, M., Attwood, J.T., Bondarev, I., Conway, S.J., Marshall, B., Brown, C., Mellor, A.L., 1998. Prevention of allogeneic fetal rejection by tryptophan catabolism. Science 281, 1191-1193.
Payne, S.G., Smith, S.C., Davidge, S.T., Baker, P.N., Guilbert, L.J., 1999. Death receptor Fas/Apo-1/CD95 expressed by human placental cytotrophoblasts does not mediate apoptosis. Biol. Reprod. 60, 1144-1150.

Raghupathy, R., 1997. Th1-type immunity is incompatible with successful pregnancy. Immunol. Today 18, 478-482.

Runic, R., Lockwood, C.J., Ma, Y., Dipasquale, B., Guller, S., 1998. Expression of Fas ligand by human cytotrophoblasts: implications in placentation and fetal survival. J. Clin. Endocrinol. Metab. 81, 3119-3122.

Senturk, L.M, Arici, A., 1998. Leukemia inhibitory factor in human reproduction. Reprod. Immunol. 39, 144-151.

Siiteri, P.K., Stites, D.P., 1982. Immunologic and endocrine interrelationships in pregnancy. Biol. Reprod. 26, 1-14.

Thellin, O., Coumans, B., Zorzi, W., Igout, A., Heinen, E., 2000. Tolerance to the foeto-placental 'graft': ten ways to support a child for nine months. Curr. Opin. Immunol. 12, $731-737$.

Xu, C., Mao, D., Holers, V.M., Palance, B., Cheng, A.M., Molina, H., 2000. A critical role for murine complement regulator Crry in fetomaternal tolerance. Science 287, 498501. 